\title{
Interactive tracking of insect posture
}

\author{
Minmin Shen ${ }^{\mathrm{a}, \mathrm{b}, *}$, Chen $\mathrm{Li}^{\mathrm{c}}$, Wei Huang ${ }^{\mathrm{d}}$, Paul Szyszka ${ }^{\mathrm{e}}$, Kimiaki Shirahama ${ }^{\mathrm{c}}$, \\ Marcin Grzegorzek ${ }^{\mathrm{c}}$, Dorit Merhof ${ }^{\mathrm{f}}$, Oliver Deussen $^{\mathrm{a}}$ \\ a Department of Computer and Information Science and the INCIDE Center, University of Konstanz, Konstanz, D-78464 Konstanz, Germany \\ ${ }^{\mathrm{b}}$ School of Software Engineering, South China University of Technology, Guangzhou 510640, China \\ ${ }^{\mathrm{c}}$ Research Group for Pattern Recognition, Institute for Vision and Graphics, University of Siegen, Siegen D-57076, Germany \\ d Department of Computer Science, Nanchang University, Nanchang 330031, China \\ e Institute of Neurobiology, University of Konstanz, Germany \\ ${ }_{\mathrm{f}}^{\mathrm{f}}$ Institute of Imaging \& Computer Vision, RWTH Aachen University, Aachen, Germany
}

Keywords:

Multiple object tracking

Active key frame selection

Interactive user correction and tracks

refinement

Insect tracking

\begin{abstract}
A B S T R A C T
In this paper, we present an association based tracking approach to track multiple insect body parts in a set of low frame rate videos. The association is formulated as a MAP problem and solved by the Hungarian algorithm. Different from a traditional track and then rectification scheme, this framework refines the tracking hypotheses in an interactive fashion: it integrates a key frame selection approach to minimize the number of frames for user correction while optimizing the final hypotheses. Given user correction, it takes user inputs to rectify the incorrect hypotheses on the other frames. Thus, the framework improves the tracking accuracy by introducing active key frame selection and interactive components, enabling a flexible strategy to achieve a trade off between human effort and tracking precision. Given the refined tracks at a bounding box (BB) level, the tip of each body part is estimated, and multiple body parts in a BB are further differentiated. The efficiency and the effectiveness of the framework are verified on challenging video datasets for insect behavioral experiments.
\end{abstract}

\section{Introduction}

The movements of body parts of harnessed insects, such as antennae or mouthparts, provide information about internal states [1], sensory processing [2] and learning [3 6]. Although there is some research reported in animal tracking, estimating the center of body mass (position) is much simpler than detecting the detailed body posture and position of appendages (pose) [7]. To the best of our knowledge, our work is the first research about tracking multiple insect body parts that are of different types. Insect posture is estimated as the tip of each body part (e.g. a bee's antennae or tongue as shown in Fig. 2).

Although the application scenario of our tracking framework addresses a particular task, the challenges to be addressed, however, characterize a generic tracking problem resulting from (1) varying number of targets, (2) incoherent motion, (3) occlus ion and merges, (4) all targets have dark appearance, similar shape and no texture and (5) long tracking gaps. Most tracking

\footnotetext{
* Corresponding author at: Department of Computer and Information Science and the INCIDE Center, University of Konstanz, Konstanz, D-78464 Konstanz, Germany. Tel: +497531 88 5108; fax: +497531884715.

E-mail address: minmin.shen@uni-konstanz.de (M. Shen).
}

frameworks assume a coherent motion, i.e. all the elementary targets move with similar average velocity over extended periods of time. However, this assumption does not hold here. A pictorial illustration is shown in Fig. 1, where a set of object detections as unordered bounding boxes (BBs) are produced by a standard moving object detector. Different colors are used here to denote the expected label for better visualization. It can be seen that a merged (see Fig. 1g) or false negative (FN) BB (see (b, i)) produces a tracking gap, which makes it unsuitable for frame by frame tracking approaches such as particle filter based algorithms [8]. As the mandibles (i.e. labels 2 and 4 ) do not provide much information for biologists, we do not track them in the case where they are merged or occluded.

The different occlusion and merge conditions are illustrated as in Fig. 2. We already attempted to address partly these issues in our previous work [9], but the targets are difficult to differentiate at a BB level under merge conditions (see Fig. 2a and e). In this application, we denote occlusion as the cases where target $a$ is occluded by target $b$, and merge where targets $a$ and $b$ are merged at the same BB. For occlusion conditions, estimating the position of an occluded target $a$ if it is not visible makes little sense, though maintaining its identity when it appears again is challenging. For merge conditions, we propose a new algorithm to differentiate 
a

b

c

d e

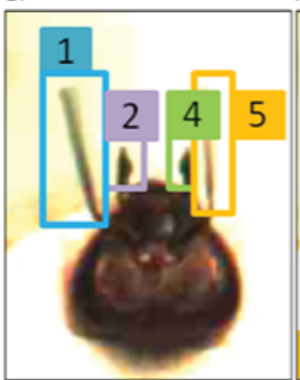

ir
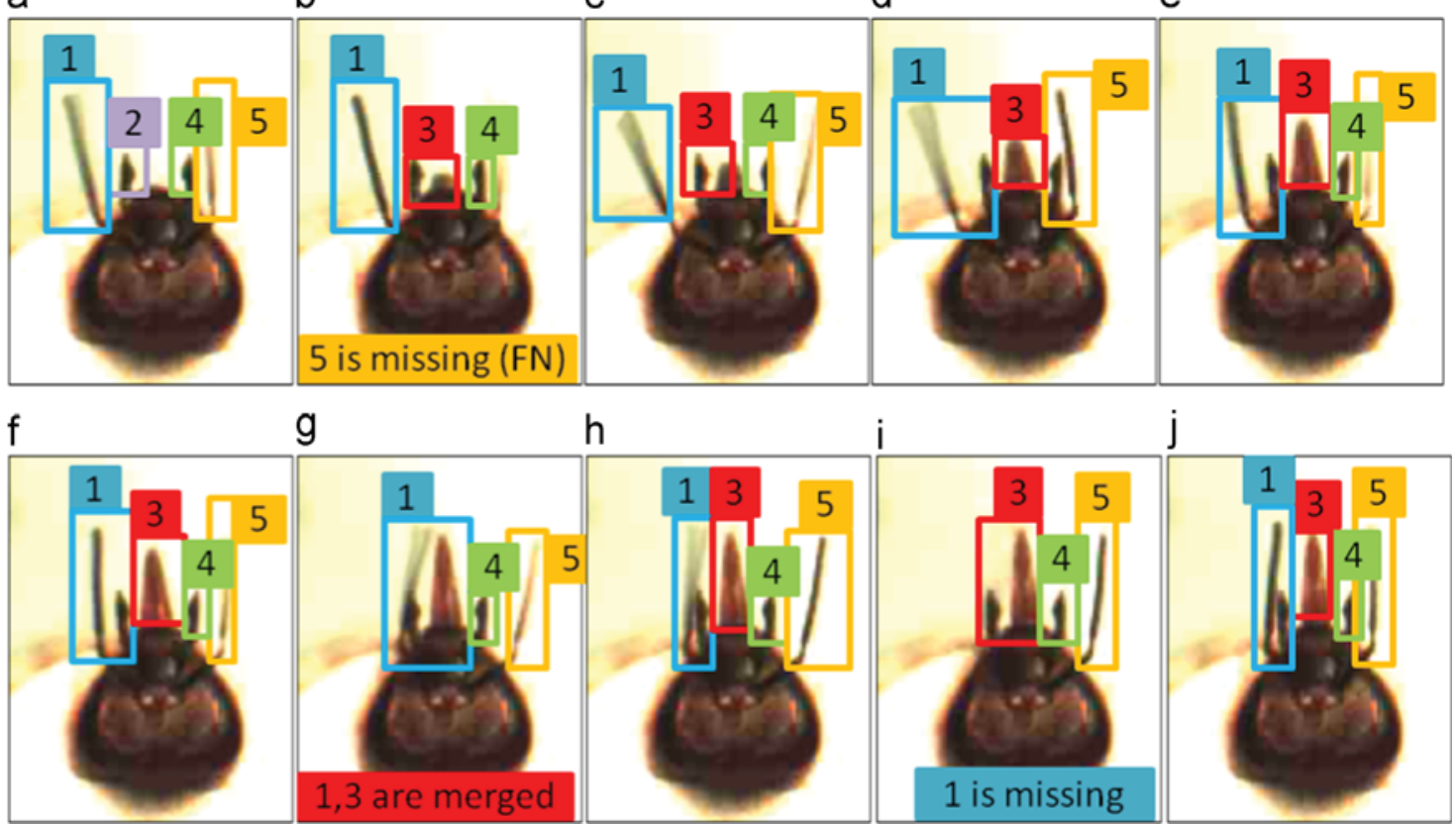

h i

i j
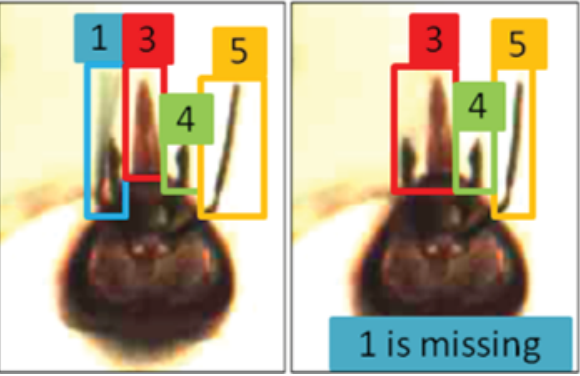

j

Fig. 1. Object detections at 10 consecutive frames including merged and false negative BBs. Identification of each BB, shown in
label for each body part is denoted as (1) left antenna; (2) left mandible; (3) proboscis; (4) right mandible; (5) right antenna.

a

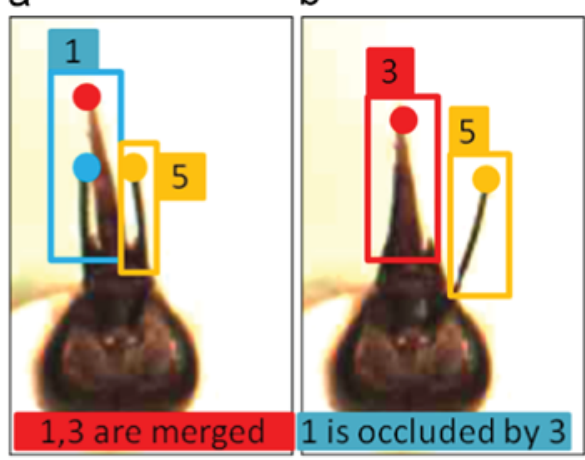

C

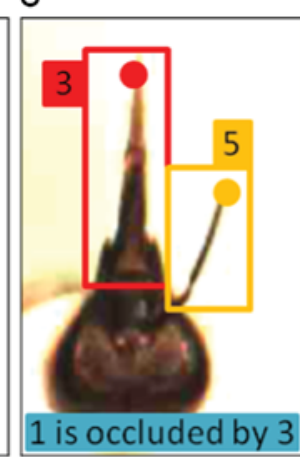

d

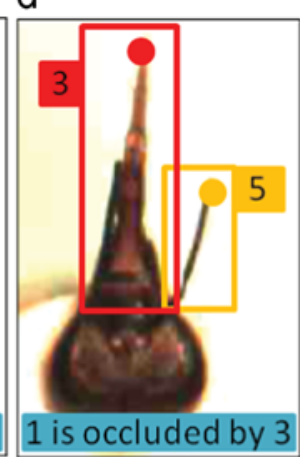

e

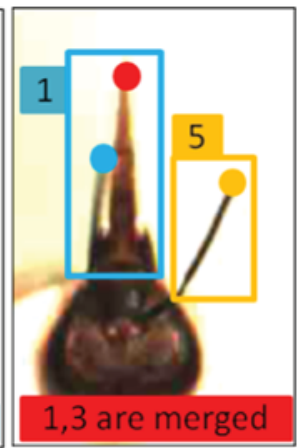

Fig. 2. Sample frame of (a, e) merge or (b, c, d) occlusion. Merged targets are difficult to be differentiated at the BB level, thus we propose to estimate the position of the tip of each target, which is denoted as a solid circle in the corresponding color. (A colored version is available online.)

targets at pixel precision by estimating the tip of each target (shown as the small solid circle in Fig. 2a and e).

The tracking problem of this paper is formulated as follows. The inputs to our tracking framework are a set of detection responses at the BB level, thus only provide rough estimation of the targets' positions. We denote the detection responses by $\mathbf{Z}_{1: N}=\left\{\mathbf{z}_{i, t} \mid\right.$ $\left.1 \leq i \leq n_{t}, 1 \leq t \leq N\right\}$, where $n_{t}$ is the number of detection responses at time $t$. Our objective is to estimate the trajectories of the tips of $n$ targets. In the case of a honey bee, $n=5$, i.e. (1) right antenna; (2) right mandible; (3) proboscis; (4) left mandible; (5) left antenna. The trajectories are denoted as $\mathbf{T}=\left\{T_{t_{11}, t_{i 2}}^{i} \mid\right.$ $1 \leq i \leq n\}$, where $T_{t_{i 1}, t_{i 2}}^{i}$ is the track of the $i$ th target existing from time $t_{i 1}$ to $t_{i 2}$.

In this paper, we propose an interactive framework for insect tracking integrating a frame query approach, instead of the traditional track and then rectification scheme. As shown in Fig. 3, the overall framework includes six stages: (1) moving object detection, (2) feature extraction, (3) classification of moving objects, (4) constrained frame to frame linking, (5) key frame (KF) estimation and annotation query and (6) track linking through merge conditions. The yellow blocks highlight the interactive part, while the blue blocks indicate the automated computation part. We will address our tracking problem by fulfilling two sub tasks. The first sub task is to assign a label $y_{i, t}$ to the corresponding $\mathrm{BB} \mathbf{z}_{i, t}$, and construct tracks at the BB level $\mathbf{Y}_{1: N}=\left\{y_{i, t} \mid 1 \leq i \leq n_{t}, 1 \leq t \leq N\right\}$. Given the input $\mathbf{z}_{i, t}$, a feature vector $\mathbf{f}_{i, t}$ is extracted to represent the information about its position, motion and shape. The initial label $y_{i, t}$ is estimated by classification (Section 4.1) and constraint frame to frame linking (Section 4.2). This framework queries users to rectify the incorrect labels only for certain frames (i.e. $Y_{S} \mid S \in \Phi$, where $\Phi$ is the set of KFs), which are estimated in Section 4.3, and the framework takes them as prior information to compute the labels of BBs on the other frames. The tracks are iteratively refined until user query is no longer required. As a result, reliable tracks $\mathbf{Y}_{1: N}$ are constructed, which is indicated with a pink shaded ellipse in Fig. 3a. The second sub task is to find the position of the tip (i.e. the endpoint, shown as colored solid circles in Fig. 2) of each target $\mathbf{x}_{t}^{i}$ and construct complete tracks $\mathbf{T}=\left\{T_{t_{11}, t_{i 2}}^{i} \mid 1 \leq i \leq n\right\}$ through merge or occlusion conditions, which are indicated as solid colored lines in Fig. 3b. We propose an algorithm in Section 4.4 to link the gaps between the tracks to compute automatically the final trajectories $\mathbf{T}$. 


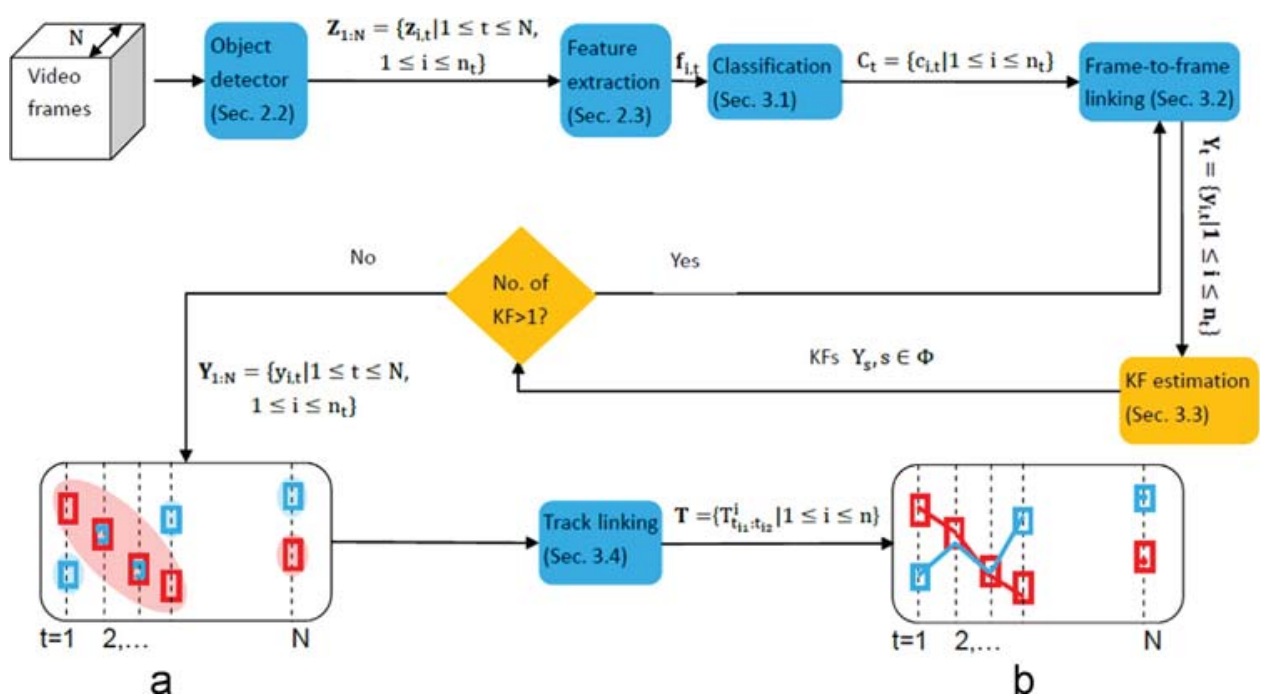

Fig. 3. The flowchart of the overall tracking framework: the yellow blocks highlight the interactive part, while the blue blocks denote the automated computation part. (A colored version is available online.)

The rest of this paper is organized as follows. The related work to this paper is summarized in Section 2. The preliminaries are introduced in Section 3, including object detection and preproces sing (Section 3.1) and an anatomical model of insect body parts (Section 3.2). The proposed tracking framework is elaborated in Section 4. Its practicability and accuracy are validated by experi mental results in Section 5. Section 6 concludes this paper.

\section{Related work}

The multiple object tracking (MOT) approaches could be classified into two categories [10]: association based tracking approaches and category free tracking. The former category usually first localizes objects in each frame and then links these object hypotheses into trajectories without any initial labeling. The latter one, also referred as online object tracking [11], requires the initialization of a fixed number of objects in the first frame (in the form of BBs or other shape configurations), then localizes these fixed number of objects in the subsequent frames. As we aim to track varying number of objects, we adopt the association based tracking approach. The success of most existing association based tracking algorithms comes from a discriminative appearance model (using the cues of color or texture) [12,13], or constant velocity motion of targets [12 15]. There are a few published studies that address problems in tracking animals. They include algorithms for tracking freely moving animals (e.g. bee dance $[16,17]$, ants $[18,19])$ and freely moving body parts of harnessed animals (e.g. bees' antennae [20], mouse whiskers [21]). A more detailed review could be found in [53]. We summarize the related work to this paper and their main characteristics in Table 1, including the tracking framework, appearance model and type or number of target(s). We also list the assumptions of these works according to the authors, which may make them inapplicable for our case. In this paper, we take an association based approach, designing a MAP framework that maps the difficult MOT problem into a simpler object classification problem with regularization via temporal correlations. This method is able to address the chal lenges here such as incoherent motion and merge or occlusion conditions.

To overcome the bottleneck of the automatic tracking perfor mance by introducing user input, some interactive algorithms have been reported [25 28]. But some of them either requires users to view the whole video $[26,25]$, or not to focus on frame query techniques [27]. The most conceptually similar work to ours is proposed in [28], which extends the tracker in [29] by estimat ing more KFs for user annotation to improve the tracking accuracy. However, since the KF estimation scheme in [28] punishes significant label change, it is not applicable in our task, where different objects could be detected in turns at the same position (see Fig. 1(f,g)).

\section{Preliminaries}

When controlled stimulus conditions are needed, insects are often restrained and their behavior is monitored as movements of body parts such as their antenna or mouthparts. The proboscis is the mouthpart of the insect and hungry bees extend their proboscises reflexively when stimulated with food or with a previously conditioned odorant (Fig. 4).

\subsection{Object detection and preprocessing}

As our interests focus on tracking the antennae and mouthparts when they are moving, it is preferred to detect the moving part rather than segmenting the body part on a single frame basis. Thus, Gaussian Mixture Model's (GMM) background modeling [30] is used. A more advanced background subtraction method based on a dynamic background model [31] may reduce false detections, but a standard moving object detector is used here as we focus on the tracking part. The object detector generates an unordered set of bounding boxes (BBs) including false positives (e.g. shadows, reflection and the insect's legs), false negatives (e.g. motion blurred antennae), missing objects (e.g. the antenna above the insect's head, or the proboscis not extended), merged detections (one bounding box including two or three objects) and occluded detections, which make the following tracking task difficult. Therefore, pre processing operations include shadow removal [30], exclusion of undesired objects by incorporating position information, and segmentation of merged measurements.

These pre processing operations greatly reduce the undesired detection measurement, but some false, missing, merged mea surements may still remain. Thus, a subsequent tracking algorithm is required to tackle this problem. 
Table 1

Related work and their main characteristics.

\begin{tabular}{|c|c|c|c|c|}
\hline Algorithm & Tracking framework & Appearance model & $\begin{array}{l}\text { Type/number of } \\
\text { target(s) }\end{array}$ & Remarks \\
\hline$[14]$ & Hungarian & Foreground response & $\begin{array}{l}\text { Multiple generic } \\
\text { objects }\end{array}$ & Assume coherent motion \\
\hline [12] & Hungarian & Color histogram & $\begin{array}{l}\text { Multiple human } \\
\text { pedestrians }\end{array}$ & Assume coherent motion \\
\hline [15] & Hungarian & Foreground response & Multiple cars & Assume coherent motion \\
\hline [13] & Hungarian & Color histogram & $\begin{array}{l}\text { Multiple human } \\
\text { pedestrians }\end{array}$ & Assume coherent motion \\
\hline [16] & Particle filter & Geometrical features & Single bee & Incorporate specific behavior model \\
\hline [17] & Particle filter & Optical flow & Single bee & Assume coherent motion \\
\hline [8] & Particle filter & Foreground response & $\begin{array}{l}\text { Multiple mice and } \\
\text { larvae }\end{array}$ & Assume coherent motion \\
\hline [18] & $\begin{array}{l}\text { Simple data association } \\
\text { technique }\end{array}$ & Foreground response & Multiple ants & Does not tackle occlusion and merges \\
\hline [19] & Particle filter & Foreground response & Multiple ants & Assume coherent motion \\
\hline$[22]$ & Not specified & Specific warm-like insect features & $\begin{array}{l}\text { Multiple Drosophila } \\
\text { larvae }\end{array}$ & $\begin{array}{l}\text { Does not resolve collisions involving more than two } \\
\text { animals }\end{array}$ \\
\hline [23] & Hungarian & Area of connected components & $\begin{array}{l}\text { Multiple Drosophila } \\
\text { adults }\end{array}$ & Assume coherent motion \\
\hline$[24]$ & Graph based framework & $\begin{array}{l}\text { Combined features that capture local } \\
\text { spatiotemporal structure }\end{array}$ & $\begin{array}{l}\text { Multiple Drosophila } \\
\text { larvae }\end{array}$ & Training samples of encounters of two larvae required \\
\hline [20] & $\begin{array}{l}\text { Antennae identified by two } \\
\text { largest clusters }\end{array}$ & None & Two bee antennae & $\begin{array}{l}\text { Does not tackle MOT problems including merge, } \\
\text { occlusion, etc. }\end{array}$ \\
\hline [21] & Probabilistic framework & Splines & Mouse whiskers & $\begin{array}{l}\text { Does not tackle MOT problems including merge, } \\
\text { occlusion, etc. }\end{array}$ \\
\hline [7] & Probabilistic framework & Intensity map & $\begin{array}{l}\text { Constant number of } \\
\text { animals }\end{array}$ & $\begin{array}{l}\text { Assume two blobs of the same object overlap in some } \\
\text { consecutive frames, etc. }\end{array}$ \\
\hline
\end{tabular}

a

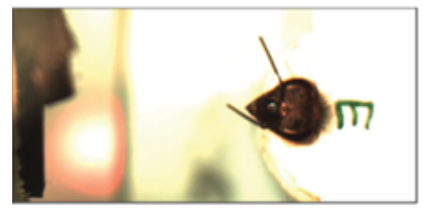

C

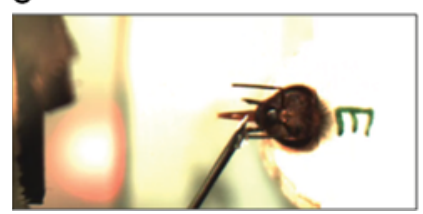

Fig. 4. Associative odor-reward learning paradigm in honey bees. A bee that has learned the association between an odorant and a food-reward extends its proboscis when stimulated with the learned odorant: (a) before odorant stimulation, (b) odorant released indicated by the LED, (c) sugar rewarding, (d) during odorant stimulation.

\subsection{Anatomical model of insect body parts}

Modeling the anatomy of an insect's head is important for accurate tracking, due to the physical limitations of the moving objects' relative positions. The positions of insect body parts (e.g. antennae and mouthparts) are ordered in a certain sequence, which is rather similar among various insects. Fig. 5 shows an image of an ant's head. These body parts are symmetric, thus they could be classified according to their types, and then further identified (tracked) by exploiting the temporal correlation between neighboring frames. Our framework incorporates an anatomical model of insects' heads as a priori, which is elaborated in Section 4.2.

We use a feature vector $\mathbf{f}_{i, t}$ to represent each BB in terms of its position, motion and shape. We follow our previous work [32,33] to extract the information of position and motion. A challenge in our tracking task results from the similarity of the objects of interest, all of which have dark appearance, similar shape, and no

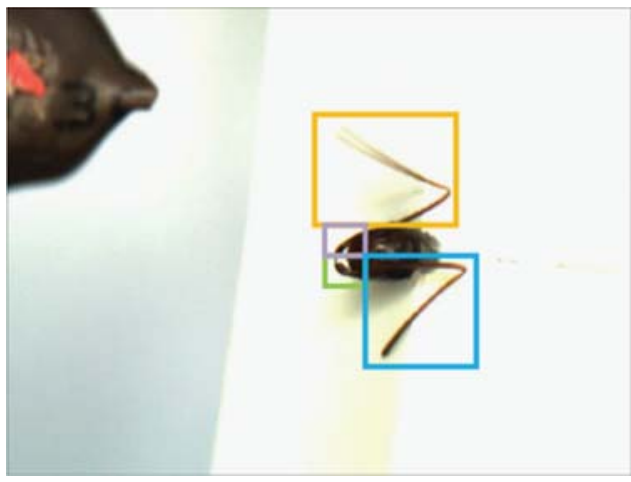

Fig. 5. The closed up sample video frame of an ant. In the case of an ant, two antennae (yellow and blue BB) and its mouthparts (purple and green $\mathrm{BB}$ ) need to be tracked (i.e. $n$ 4). (A colored version is available online.)

texture. Therefore, some widely used features (such as color histogram [34], image patch [27] and Haar like features [35]) are not good choices for discriminative representation here. For example, the advantage of point based features originates from the discriminative local appearance at interest points [36 38], which is distinct from surroundings (or other targets) and remains consistent over time. However, the local features at interest points of our targets vary dramatically over time, as they tend to move incoherently. It is illustrated in Fig. 6 where the Kanade Lucas Tomasi (KLT) feature tracker [39] fails to track the left antenna. The initial interest points are detected by a corner detector [40].

To characterize the shape of each object, an appropriate shape descriptor should be used to model its appearance. The appear ance model based on shape context has been successfully used in many machine vision tasks such as frontal face recognition [41], smooth object retrieval [42]. We used the top hat filter as a line detector in our previous work [32] to differentiate a bee's antenna from other objects, as a bee's antenna is line shaped. But this is not applicable for the other insects such as ants. Popular shape descriptors include the Edge Histogram Descriptor (EHD) [43], the 
a

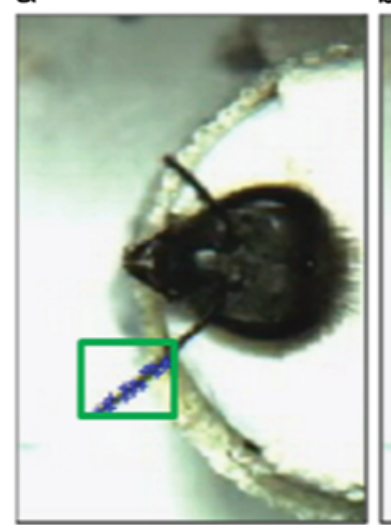

b

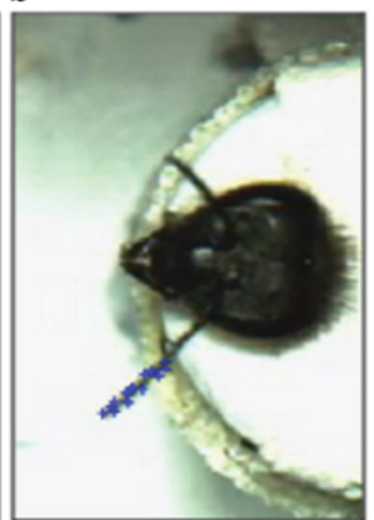

C

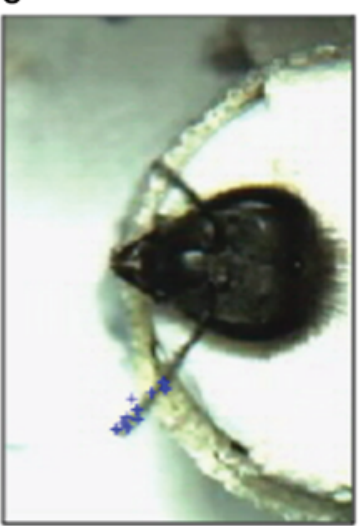

d

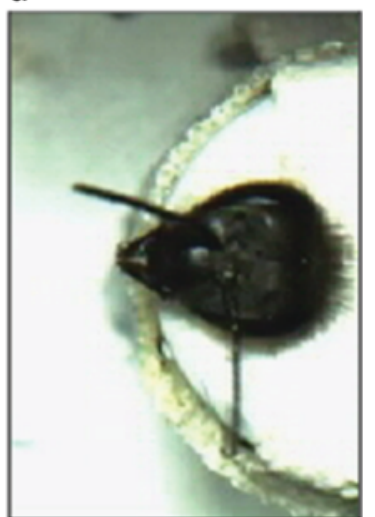

Fig. 6. The initial interest points in (a) (denoted by blue stars) are detected by corner detector within the green bounding box. The number of successfully tracked points reduces dramatically over time (b) ten, (c) six, (d) zero. (A colored version is available online.)

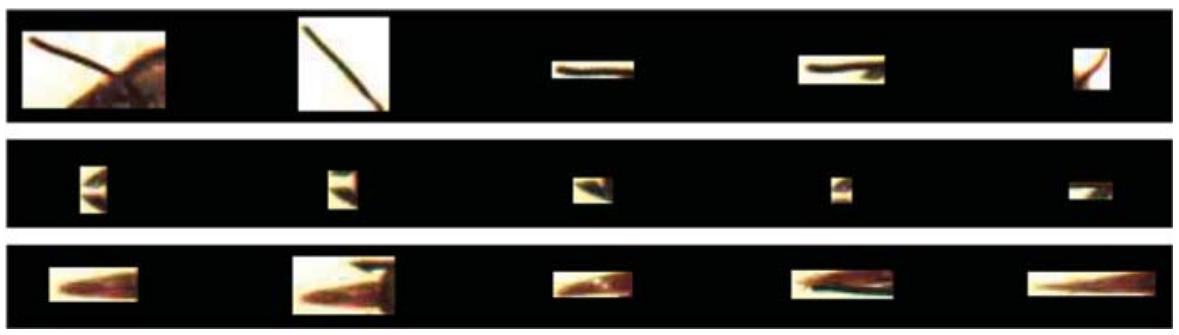

Fig. 7. The first row is detected antennae, the second row is detected mandibles and the third row is detected proboscis.

Isomerous Edge Histogram Descriptor (IEHD) [44], the Geometrical Feature (GF) [45] (including the object perimeter, area, etc.), the Shape Signature Histogram (SSH) [46], the Fourier Descriptor (FD) [47] and the Internal Structure Histogram (ISH) [44]. These six feature extraction methods describe shapes from different perspectives.

To select an effective shape feature for representing the insect body parts, two characteristics should be considered here: first, all body parts have small sizes in the frames (about 200600 pixels); second, all body parts have simple bendability, i.e. few local boundary information such as curvature and junctions are present. Some examples of detected body parts are illustrated in Fig. 7. The first characteristic makes the discrete points on the edges of the body parts limited (about 50200 points), so that the boundary based shape descriptors are not able to obtain enough good sample points. The FD also suffers from this fact. The second characteristic weakens the descriptive power of GF. We found that insect body parts' shapes embody good linear edges in different orientations. This indicates that edges are important low level features in image description, thus we choose EHD as the shape descriptor. It is verified by the comparison of the six shape descriptors in Section 5.2.3. EHD is one of the most popular edge based features, and able to describe both local and global features. In our work, EHD is used to describe the global shape features of insect body parts by two steps. First, the regional edge histograms are extracted based on five categories of edge direc tions: $45^{\circ}, 90^{\circ}, 135^{\circ}, 180^{\circ}$ and any other degrees. Second, a global edge histogram is calculated as the mean value of the extracted histograms.

\section{Proposed interactive framework}

Similar to many association based approaches (e.g. [48]), we define the association as a MAP problem. Our objective is to determine correspondence of multiple BBs through $N$ frames. Under the MAP framework, a global optimum $\widehat{\mathbf{Y}}_{1: N}$ is found by maximizing the posterior probability $P\left(\mathbf{Y}_{1: N} \mid \mathbf{Z}_{1: N}\right)$ :

$\widehat{\mathbf{Y}}_{1: N}=\arg \max _{\mathbf{Y}_{1: N}} P\left(\mathbf{Y}_{1: N} \mid \mathbf{Z}_{1: N}\right)=\arg \max _{\mathbf{Y}_{1: N}} \prod_{t}^{N} P\left(Z_{t} \mid Y_{t}\right) P\left(\mathbf{Y}_{1: N}\right)$

where $Z_{t}$ and $Y_{t}$ are ordered collections of BBs $\mathbf{z}_{i, t}$ and its label $y_{i, t}$ at time $t: Z_{t}=\left\{\mathbf{z}_{i, t} \mid 1 \leq i \leq n_{t}\right\}, Y_{t}=\left\{y_{i, t} \mid 1 \leq i \leq n_{t}\right\} . P\left(Z_{t} \mid Y_{t}\right)$ is the likelihood that the collection of BBs $Z_{t}$ is generated from the sequence of labels $Y_{t}$. We assume that $Y_{t}$ is temporal independent of each other. $P\left(\mathbf{Y}_{1: N}\right)$ is the a priori probability of a labeling sequences $\mathbf{Y}_{1: N}$. The labels are initially estimated at the frame level (Section 4.1), and then temporal correlation is considered for refinement by data association (Section 4.2).

\subsection{Object classification}

Due to the symmetry of insect's appearance, a detection response $\mathbf{z}_{i, t}$ is first classified as one of $m$ classes $c_{i, t}$, where $c_{i, t} \in\{1:$ antenna; 2 : mandible; 3 : proboscis $\}$. Its label $y_{i, t}$ is esti mated by differentiating the details (either on the left hand side or the right hand side) in the following tracking step.

In this paper, we select the Support Vector Machine (SVM) as a classifier. It improves the performance of our previous work in [9] due to its advantage of dealing with high dimensional data. Probability based classifiers (Naïve Bayes) need a large number of training examples to appropriately estimate probabilistic dis tributions in high dimensional feature spaces $[9,49]$. Similarity based classifiers (e.g. $k$ Nearest Neighbor) fail to appropriately measure similarities in high dimensional feature spaces, because of many irrelevant dimensions. In this work, we adopt a multi class Support Vector Machine (mSVM) using the one against one (1vs1) strategy. Each class is determined by computing pair wise votes using two class SVMs. In the case of $K$ classes, $K\left(\begin{array}{ll}K & 1\end{array}\right) / 2$ two class classifiers are trained. The final classification result is 


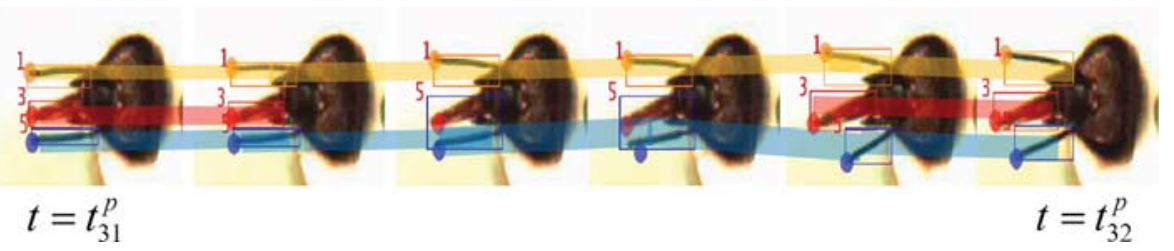

Fig. 8. An example of linking tracks through merge condition: the shaded lines indicate the tracks at the BB level and the circles indicate the tips. $t_{31}^{p}$ and $t_{32}^{p}$ indicate the tail and head of the $p$ th tracklet of the proboscis (i.e. label 3) $T_{t_{31}^{p}, t_{32}^{p}}^{3}$, respectively.

determined by counting which class the object has been assigned to most frequently.

The object classification generates a class label $c_{i, t}$ and the corresponding class probability $P\left(c_{i, t} \mid \mathbf{z}_{i, t}\right)$ for each BB. Given the output of this classification step, however, two challenges remain in the following tracking task: (1) incorrect classification hypoth eses and (2) identity swapping due to the interaction of moving objects.

\subsection{Constrained frame to frame linking}

Based on the output of object classification $c_{i, t}$, we exploit the appearance information of an insect, i.e. position and ordering of $\mathbf{z}_{i, t}$, to assign the label $y_{i, t}$. As we assume that the likelihood $P\left(Z_{t} \mid Y_{t}\right)$ is temporally independent, the label $y_{i, t}$ is determined by the class label $c_{i, t}$ and the relative position of $\mathbf{z}_{i, t}$ to the origin (left or right).

Incorporating prior knowledge of the appearance model: The likelihood $P\left(Z_{t} \mid Y_{t}\right)$ is estimated following the constraint that $Z_{t}$ should be ordered in an ascending manner, as insect body parts are assumed to be ordered in a certain sequence. The label sequences $Y_{t}$ that violate this assumption will be considered as incorrect hypotheses (i.e. $P\left(Z_{t} \mid Y_{t}\right)=0$ ). For other $Y_{t}$, the likelihood $P\left(Z_{t} \mid Y_{t}\right)$ is computed considering the rule of combination without repetition, as $n_{j}$ BBs are detected out of $n$ objects.

$P\left(Z_{t} \mid Y_{t}\right)= \begin{cases}0 & \text { if } \hat{m}_{1}>m_{1} \text { or } \hat{m}_{2}>m_{2} \\ & \text { or } \hat{m}_{3}>m_{3} \text { or } \exists \mathbf{z}_{i, t}>\mathbf{z}_{k, t}, \forall k<i \\ \left(\begin{array}{c}n \\ n_{j}\end{array}\right) & \text { otherwise }\end{cases}$

where $m_{k}$ is the number of $\left\{C_{t} \mid c_{i, t}=k\right\}$. This is considered as a priori knowledge incorporating the characteristics of insects' appearance. It is easily adapted to other insects by setting the value of $m_{k}$ and $n$.

Estimation of benchmark frames: The frames with the highest posteriori probabilities are assumed to be correct hypotheses. Among these frames, we define a set of frames $\Psi$ as the benchmark frames: $Y_{b}$, where $b \in \Psi: P\left(Z_{t} \mid Y_{t}\right)=1 \& P\left(Z_{t \pm 1} \mid Y_{t \pm 1}\right) \neq 1$.

We define $P\left(\mathbf{Y}_{1: N}\right)$ in (1) to guarantee that only the benchmark frames are used to help rectify the potentially incorrect hypoth eses on their neighboring frames by data association:

$P\left(\mathbf{Y}_{1: N}\right)=\prod_{b \in \Psi} P\left(Y_{b \pm 1} \mid Y_{b}\right)$

The conditional probability $P\left(Y_{b \pm 1} \mid Y_{b}\right)$ is defined as a function of the pairwise linking cost between $Y_{b}$ and $Y_{b \pm 1}$ :

$P\left(Y_{b \pm 1} \mid Y_{b}\right)=\prod_{i, k} P\left(y_{i, b} \mapsto y_{k, b \pm 1}\right)$

where the sign “ $\mapsto$ " denotes correspondence. The frame to frame linking between $Y_{b}$ and $Y_{b \pm 1}$ is found by forming an $n_{t} \times n_{t}$ cost matrix $\mathbf{M}=\left\{M_{i, k}\right\}$ with

$M_{i, k}=\log P\left(y_{i, b} \mapsto y_{k, b \pm 1}\right)=\left\|\mathbf{z}_{i, b} \quad \mathbf{z}_{k, b \pm 1}\right\|$

where $n_{t}=\max \left(n_{b}, n_{b \pm 1}\right)$ and the sign “ $\mapsto$ " denotes correspon dence. As an association optimization algorithm, Hungarian algorithm [50] is applied to find the optimal linking by minimizing the linking cost.

The likelihood of frames $Y_{b \pm 1}$ (i.e. those frames that are rectified with $Y_{b}$ ) is recomputed as

$P\left(Z_{b \pm 1} \mid Y_{b \pm 1}\right)= \begin{cases}0 & \text { if } \hat{m}_{1}>m_{1} \text { or } \hat{m}_{2}>m_{2} \\ \text { or } \hat{m}_{3}>m_{3} \text { or } \exists \mathbf{z}_{i, b \pm 1}>\mathbf{z}_{k, b \pm 1}, \quad \forall k<i \\ 1 \quad \text { otherwise }\end{cases}$

New benchmark frames are estimated and frame to frame linking is performed iteratively.

\subsection{KF estimation and annotation query}

According to Eqs. (1) and (3), $\widehat{\mathbf{Y}}_{1: N}$ is the current optimal estimation for the labels given a set of benchmark frames in $\left\{Y_{b}, b \in \Psi\right\}$ estimated in Section 4.2. The success of frame to frame linking lies in the estimation of benchmark frames. We use prior knowledge in (2) to initially estimate the set of benchmark frames $\Psi$, but the constraints in (2) do not always hold, and some frames could not be rectified with the given benchmark frames.

To refine further $\widehat{\mathbf{Y}}_{1: N}$, it is required to determine new bench mark frames $\left\{Y_{b}, b \in \Psi\right\}$ in (3) to form a new set $\Psi^{*}$ by introducing human effort. With the new benchmark frames, the constraint in (2) is relaxed. To minimize user effort, we propose an approach to minimize the number of KFs while optimizing the final hypothesis. The intuitive concept is that only the potential benchmark frames should be rectified, so that corrections on the rectified KFs could propagate to their neighboring frames in the subsequent frame to frame linking. Given the new set $\Psi^{*}$ with added KFs obtained from the user annotation, we combine Eqs. (1) and (3) and define a new cost function

$\widehat{\mathbf{Y}^{*}}{ }_{1: N}=\arg \max _{\mathbf{Y}_{1: N}^{*}} \prod_{t}^{N} P\left(Z_{t} \mid Y_{t}\right) \prod_{b \in \Psi^{*}} P\left(Y_{b \pm 1} \mid Y_{b}\right)$

The refined labels $\widehat{\mathbf{Y}^{*}}$ 1:N are found by solving (7).

As illustrated in Fig. 3, we refine the incorrect hypotheses in $\widehat{\mathbf{Y}}_{1: N}$ by interactively (1) requesting user correction on estimated KFs; (2) taking corrected KFs and rectifying their neighboring frames by frame to frame linking; and (3) updating KFs. We define the annotation cost of each frame to indicate the degree of "usefulness" of user annotation, in order to estimate which frame should be the potential benchmark frame and added to form a new set of benchmark frames $\Psi^{*}$. The higher the annotation cost is, the more erroneous $Y_{t}$ tends to be. Naturally, the annotation cost is related to the probability of incorrect hypothesis. Here we consider two conditions of frames $\widehat{\mathbf{Y}}_{1: N}$, i.e. $Y_{b \pm 1}$ and the others. For $Y_{b \pm 1}$, we should also take their association with $Y_{b}$ into consideration. Therefore, the annotation cost is defined as

$A\left(Y_{t}\right)=P_{\epsilon}\left\{\begin{array}{lll}1 & P\left(Z_{t} \mid Y_{t}\right) \prod_{i, k} P\left(y_{i, t} \mapsto y_{k, t \pm 1}\right), & t=b \pm 1 \\ 1 & P\left(Z_{t} \mid Y_{t}\right) & \text { otherwise }\end{array}\right.$

As $A\left(Y_{t}\right)$ interprets the probability that $y_{i, t}$ could be an incorrect hypothesis, it provides a flexible strategy for users to set the threshold $\tau$, for which one could choose KFs from the frames $A\left(Y_{t}\right) \geq \tau$ considering the trade off between tracking accuracy and 
human effort. The KFs $Y_{s}$ are defined as $s \in \Phi: P\left(X_{S-1} \mid Y_{S} \quad 1\right)=$ $1 \& A\left(Y_{s}\right) \geq \tau$. Users are queried to rectify the KFs $Y_{s}$, which are subsequently used to form a new set of benchmark frames as $\Psi^{*}=\Psi \cup \Phi$.

\subsection{Track linking through merge conditions}

Given reliable tracklets $\widehat{\mathbf{Y}^{*}}{ }_{1 \cdot N}$ as benchmarks, we treat them as rough approximation of the tips. To extract further the positions of the tips of each object at the pixel level $\mathbf{x}_{t}^{i}$ through merge conditions, we propose an approach to link the tracklets by interpolating the missing tracklets on the in between frames. Let us denote the track of the $i$ th target as a set of tracklet association $T_{t_{11}^{p}, t_{i 2}^{p}}^{i}=\left\{\mathbf{x}_{t}^{i} \mid t_{i 1}^{p} \leq t \leq t_{i 2}^{p}\right\}$, where $t_{i 1}^{p}$ and $t_{i 2}^{p}$ indicate the tail and head of the $p$ th tracklet of $T_{t_{i},}^{i}, t_{i,}^{p}$, respectively. An example of linking tracks through merge condition is illustrated in Fig. 8.

For the merge condition where tips of targets $a$ and $b$ are merged (i.e. they are bounded within the same BB labeled $y_{a, t}$ ), we define $P_{m}\left(\mathbf{x}_{t}^{a}, \mathbf{x}_{t}^{b} \stackrel{m}{\rightarrow} y_{a, t}\right)$ to indicate the probability of merge. It is defined as the product of the independent appearance component $P_{a, m}\left(\mathbf{x}_{t}^{a}, \mathbf{x}_{t}^{b} \stackrel{m}{\rightarrow} y_{a, t}\right)$ and the temporal component $P_{t, m}\left(\mathbf{x}_{t}^{a}, \mathbf{x}_{t}^{b} \stackrel{m}{\rightarrow} y_{a, t}\right)$, respectively.

$P_{m}\left(\mathbf{x}_{t}^{a}, \mathbf{x}_{t}^{b} \stackrel{m}{\rightarrow} y_{a, t}\right)=P_{a, m}\left(\mathbf{x}_{t}^{a}, \mathbf{x}_{t}^{b} \stackrel{m}{\rightarrow} y_{a, t}\right) P_{t, m}\left(\mathbf{x}_{t}^{a}, \mathbf{x}_{t}^{b} \stackrel{m}{\rightarrow} y_{a, t}\right)$

where

$P_{a, m}\left(\mathbf{x}_{t}^{a}, \mathbf{x}_{t}^{b} \stackrel{m}{\rightarrow} y_{a, t}\right)= \begin{cases}1 & \text { if } \mathbf{f}_{a, t} \in \Xi \\ 0 & \text { otherwise }\end{cases}$

$P_{t, m}\left(\mathbf{x}_{t}^{a}, \mathbf{x}_{t}^{b} \stackrel{m}{\rightarrow} y_{a, t}\right)= \begin{cases}1 & \text { if } t_{b 1}^{p}<t<t_{b 2}^{p} \\ 0 & \text { otherwise }\end{cases}$

Here, $\Xi$ is a set of $\mathbf{f}_{a, t}$ that constrains the position and size of the target. The starting and ending time indices $t_{i 1}^{p}, t_{i 2}^{p}$ of the $p$ th track $T_{t_{11}^{p}, t_{i 2}^{p}}^{i}$ are empirically set by defining the gap between its temporal neighboring tracks larger than a threshold $\alpha$, i.e. $t_{i 2}^{p}<t_{i 1}^{p+1} \alpha$.

We initialize the estimated tracks as the set of confident tracklets $\mathbf{T}^{0}=\left\{\mathbf{x}_{t}^{a} \mid P_{m}\left(\mathbf{x}_{t}^{a}, \mathbf{x}_{t}^{b} \stackrel{m}{\rightarrow} y_{a, t}\right)=0\right\}$. The tip $\mathbf{x}_{t}^{a}$ is determined by applying Morphological operations: the object is firstly thinned to lines, and the furthest end point to the centroid of the insect's head is estimated as the tip.

To fill the frame gap under merge condition, we use Harris corner detector to find $M$ candidate pixel positions $\mathbf{x}_{t}^{i}, 1 \leq i \leq M$ to interpolate detection responses for estimating $\mathbf{x}_{t}^{a}$ and $\mathbf{x}_{t}^{b}$. We denote the set of candidate points as $\left\{\mathbf{x}_{t}^{i} \in \Omega\right\}$. The estimated tracks are constructed with new added points that are selected from $\Omega$, which have the least pairwise linking costs to their temporal nearest neighbors in $\mathbf{T}^{0}$.

In summary, an overview of the algorithm is shown in Algorithms 1 and 2.

Algorithm 1. Summary of the proposed algorithm (Sub task 1).

Assign $y_{i, t}$ for each $\mathbf{z}_{i, t}$

Input $\left\{\mathbf{z}_{i, t}\right\}, n, m_{k}$

1. Initialization: For each frame $Z_{t}$, compute $P\left(Z_{t} \mid Y_{t}\right)$

following (2).

2. Updating:

while $\exists Y_{t} \overline{\text { updated }}$ do

end while

for $t=1, \ldots, N$ do

end for

- Find the benchmark frames $\left\{Y_{b}\right\}$, where

$b \in \Psi: P\left(Z_{t} \mid Y_{t}\right)=1 \& P\left(Z_{t+1} \mid Y_{t+1}\right) \neq 1$.

- Apply pair wise linking only on $\left\{Y_{b}, b \in \Psi\right\}$ and their temporal neighbors $Y_{b+1}$, update labels $Y_{b+1}$.

- Mark $Y_{b}, Y_{b+1}$ updated.

3. KF estimation and annotation query:

- Query user correction and receive correction

$Y_{s}, s \in \Phi: P\left(Z_{s-1} \mid Y_{s-1}\right)=1 \& A\left(Y_{s}\right) \geq \tau$.

- Form a new set of benchmark frames as $\Psi^{*}=\Psi \cup \Phi$.

- Update $P\left(Z_{s} \mid Y_{s}\right)=1, \forall s \in \Phi$.

4.

if $\Phi \neq \oslash$ then

repeat step 23

end if

Output $\widehat{\mathbf{Y}}_{1: N}, A\left(Y_{t}\right)$

Algorithm 2. Summary of the proposed algorithm (Sub task 2).

Find the tip position $\mathbf{x}_{t}^{i}$ and link tracks through merge conditions

1. Initialization:

- Construct initial tracks $\mathbf{T}^{0}=\left\{\mathbf{x}_{t}^{i} \in \Omega\right\}$.

- Estimate $t_{i 1}^{p}, t_{i 1}^{p}$ as the tail and head of the $p$ th track $T_{t_{11}}^{p}$ by empirically setting a threshold of the gap between neighboring tracks $\alpha$, i.e. $t_{i 2}^{p}<t_{i 1}^{p+1} \alpha$.

2. Updating:

for $t=t_{i 1}^{p}, \ldots, t_{i 2}^{p}$ do

if $\exists \mathbf{x}_{t}^{i} \in \Omega$ at time $t$ then

for $\epsilon=1,+1, \ldots, \quad \alpha,+\alpha$ do

if $\exists \mathbf{x}_{t+\epsilon}^{a}$ or $\mathbf{x}_{t+\epsilon}^{b} \in \mathbf{T}^{0}$ at time $t+\epsilon$

- Set $\mathbf{x}_{t+\epsilon}^{a}, \mathbf{x}_{t+\epsilon}^{b}$ as the nearest temporal neighbors.
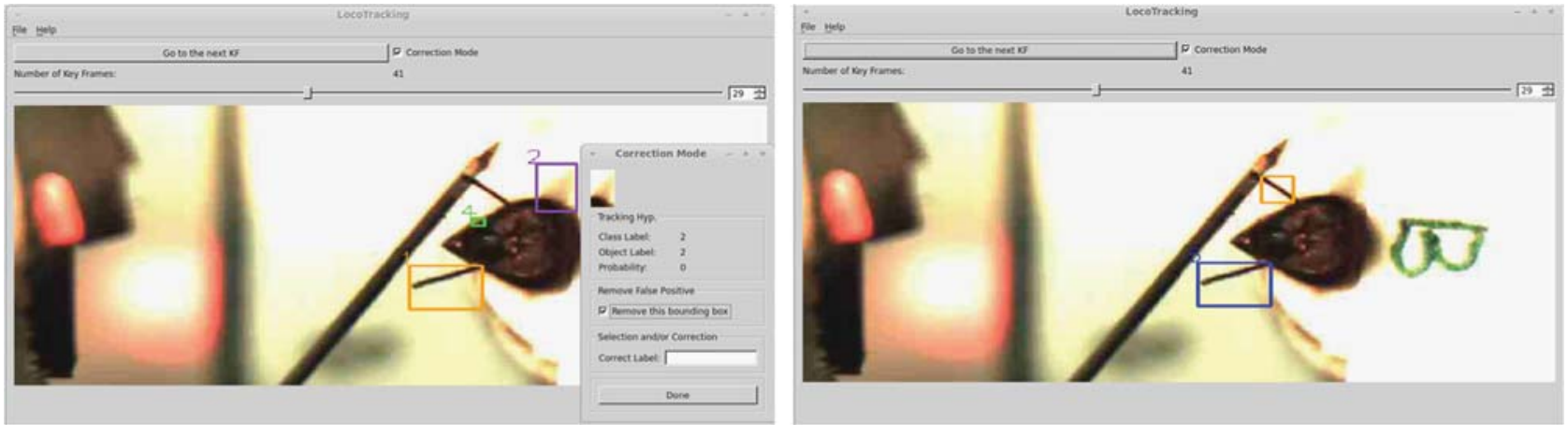

Fig. 9. Two snapshots of the GUI: initial tracking hypotheses on a KF (left) and user corrected labels (right). LocoTracker enables users to correct tracking errors including mismatches, false positives and false negatives. 
- Apply pair wise linking only on $\left\{\mathbf{x}_{t}^{i} \in \Omega\right\}$ and their nearest temporal neighbors $\mathbf{x}_{t+\epsilon_{a}}^{a}, \mathbf{x}_{t+\epsilon_{b}}^{b} \in \mathbf{T}^{0}$, determine $\mathbf{x}_{t}^{a}, \mathbf{x}_{t}^{b}$.

- Update current tracks $\mathbf{T}$ by $\mathbf{T}=\mathbf{T}^{0} \bigcup\left\{\mathbf{x}_{t}^{a}, \mathbf{x}_{t}^{b}\right\}$.
end if
end for
end if
end for

Output $\widehat{T}$.

\section{Experiments}

\subsection{Experimental setup}

Each individual insect was imaged using a CCD camera ("FMVU 03MTM/C" point gray), in order to record the head with appended body parts (e.g. proboscis, mandibles and antennae). Stimulus delivery (odor) is monitored by lighting an LED within the field of view of the camera, so that data analysis can be done relatively to stimulus delivery (see Figs. 4 and 5). Individual bees are harnessed on a platform, with their heads in fixed positions, but able to move antennae and mouthparts freely. The camera is focused statically on the top of an individual bee. Although it would be possible to record with a high speed camera, we aim at developing a framework that uses affordable cameras such as web cam or consumer level cameras, which keeps the data volume low.

We developed a system LocoTracker to implement our algo rithm in $\mathrm{C}++$, using OpenCV library version 2.4.8 (http://www. opencv.org) and tested on an Intel Core2 CPU, $3.00 \mathrm{GHz}$, with $8 \mathrm{~GB}$ RAM. We constructed a Qt based (http://qt project.org/) graphical user interface (GUI) to display KF and take user annotation in order to implement user interaction in Section 4.3. For determining the KFs, the threshold of annotation cost is set as $\tau=1$. The GUI displays each KF and the initial hypotheses, so that the user is able to recognize the errors and correct the mismatches, false negatives and false positives. Fig. 9 shows two snapshots of the GUI, illustrating how this system facilitates user interactions.

We test LocoTracker on recorded videos of two types of insects, i.e. ten videos of a bee and one video of an ant. The anatomical

Table 2

The characteristics of tested videos.

\begin{tabular}{llllll}
\hline Insect & Length & Imaging Res. $(\mathrm{pix} / \mu \mathrm{m})$ & Framerate $(\mathrm{f} / \mathrm{s})$ & GT & UO \\
\hline Bee & 8222 & 39 & 60 & 5 & $0.50 \pm 0.11$ \\
Ant & 430 & 22 & 30 & 4 & 0.13 \\
\hline
\end{tabular}

a

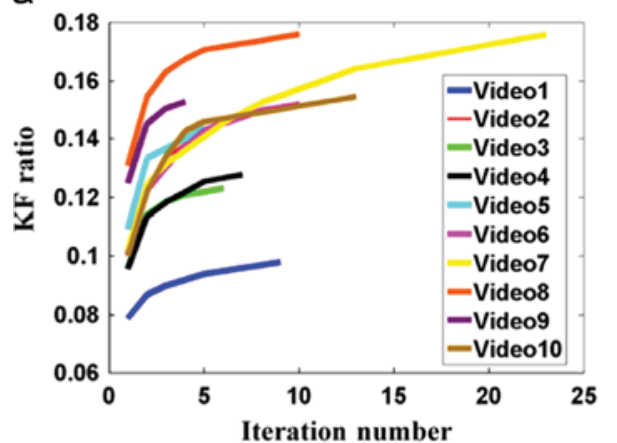

model is trained on 10 manually annotated objects for each type. The characteristics of tested videos are listed in Table 2, including length (number of tested frames), imaging resolution (pixels per $\mu \mathrm{m}$ ), frame rate (frames per second), GT (number of ground truth tracks) and UO (unobserved objects). Particularly, the ratio of UO is measured as no. of frames that contain unobserved objects/ no . of frames to indicate the tracking gaps due to complicated motion patterns of body parts (e.g. the antenna above the insect head, or the proboscis not extended). The higher the value is, the more tracking gaps the video presents.

\subsection{Experimental results}

LocoTracker is tested in terms of practicability and accuracy. We measure the practicability in two ways: (1) processing time of automated computation and user correction and (2) the trade off between human effort and tracking accuracy. Regarding accuracy, results of our algorithm are compared with some state of the art tracking methods as well as ground truth. Ground truth is manually annotated by a student in our group.

\subsubsection{Practicability}

The complexity of the algorithm is measured by processing time. We record the average running time for automated compu tation parts (Sections 3.1, 3.2, 4.1, 4.2) and the user correction time. For the running time, it takes about $0.1 \mathrm{~s}$ per frame. For recording the user correction time, the other student tested LocoTracker and it takes about $8 \mathrm{~s}$ to correct all object labels on each KF. The average of user correction time over the whole video is about $0.8 \mathrm{~s}$ per frame, thus the additional human labor is tolerant. At each iteration given the user correction for requested KFs, computing (7) takes less than $0.1 \mathrm{~s}$. Therefore, the response time of the software between two consecutive user corrections is trivial. For comparison, we tested the established software Zoo tracer [51], which also provides user correction, on bee videos. It is designed based on the prior that the displacement between adjacent frames is small and the appearance gradually changes [27]. It is a single target tracker, which takes about $6 \mathrm{~s}$ per object per frame, as user correction is required for most of the video frames.

The trade off between human intervention and tracking accu racy is tested on bee videos. Fig. 10a shows the convergence of the iterative KF estimation and annotation query (Section 4.3). The KF ratio $(K F$ ratio $=$ no. of $K F s / n o$. of frames) depends on the difficulty of tracking: more KFs are estimated for more challenging videos. For all tested videos, the main workload concentrates in the first 5 iterations. Fig. 10b shows the accuracy improvement versus the average annotation time at the 0th (before user correction), 1st, 3rd and final iteration. The accuracy is measured as the ratio of

b

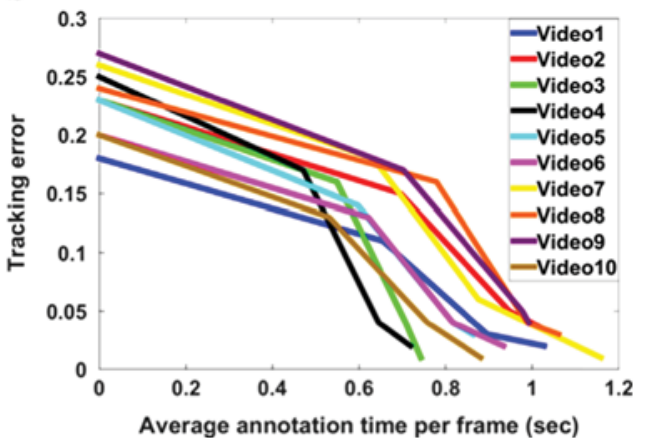

Fig. 10. (a) KF ratio vs. Iteration number of ten tested videos: the user query stops at a KF ratio of $0.1-0.18$, and the KF ratio drops dramatically within five iterations. (b) Tracking error vs. annotation time: the TE of all bee videos drops below 0.05 at the final interation, while additional annotation time is about $1 \mathrm{~s}$ on each frame. 
tracking errors TE (i.e. the number of incorrectly labeled frames) defined in [52]. The TE for all bee videos drops below 0.05 at the final interaction, while additional annotation time is about $1 \mathrm{~s}$ on each frame. In summary, the TE is $0.02 \pm 0.01$ for all tested videos, with the user correction only at the $\mathrm{KF}$ ratio as $0.14 \pm 0.02$ and additional annotation time.

\subsubsection{Accuracy}

Sub task 1: We compare our tracking method with several state of the art association based tracking and category free tracking methods.

First, our method is compared with the established software Ctrax [23] and our base tracker [33] that estimates assignment automatically. Ctrax is designed for tracking multiple Drosophila adults, but cannot tackle the situations when the number of target is not constant and if occlusions are too complex [53]. Identity switch errors occur in the cases of false detection, presence of proboscis and occlusions or merges. We tested three different methods on one of the bee videos and an ant video for compar ison. Ctrax is not applicable for tracking ant's antennae, as they do not fit the shape prior of Ctrax. The output of the bee video by Ctrax contains only the tracks of two antennae, and assumes errors in tracking other body parts, thus only these two targets are taken into account in Table 3.

Second, we tested the state of the art category free tracking methods (CT [35], MTT [54], SPOT [55] and TLD [56]) and ours on the same video. The tested codes are provided by the authors. With the initial annotated right (orange colored) and left antenna (blue colored), the tracking results at frames $\{3,11,43\}$ for first three methods and ours are shown in Fig. 11. The different tracking methods are denoted using different line types. All the compared methods start to drift from the right antenna at frame 3 , and lose both antennae at frame 43 , even when no interaction of targets presents. TLD fails to track the right antenna at the second frame, because the number of valid feature points drop from 100 to 8 . Besides, the median of forward backward error is too large (70 pixels). Its detector outputs two BBs with similar confidence, so it terminates both tracking and detection in the following frames. This indicates that category free tracking methods are

Table 3

TE of three methods on different insect videos.

\begin{tabular}{llll}
\hline Insect & Ctrax [23] & Base tracker [33] & Ours \\
\hline Bee & 0.73 & 0.10 & 0.02 \\
Ant & $\backslash$ & 0.14 & 0.02 \\
\hline
\end{tabular}

not applicable for tracking insect body parts from a low frame rate video, as temporal correlation is too weak to predict the position of target at the current frame given the previous frame.

Sub task 2: The final tracking results are the position of the tip of each object. Table 4 shows results for various flavors of our algorithm. To further show the robustness of our method, we list the ratio of detection errors after preprocessing described in Section 3.1, including merged detections, occluded detections, false negatives (FN) and false positives (FP). It is seen that the estimated positions of tips by our approach are very close to the ground truth. The mean of position error of all objects is merely 58 pixels, which are small compared to the average size of the bee's head (the size of Fig. $2 \mathrm{a}$ is $180 \times 280$ pixels). The exact position of the tip of the ant's antennae is ambiguous, because the motion blur is more severe (see the right antenna in Fig. 5a) due to a lower frame rate.

To show how well the tracks are linked, we follow [15] to use the track completeness factor TCF as measurement. A TCF of 1 is the ideal indication that the final tracks completely overlap with the ground truth. The TCF for most objects are above 0.93, except for the proboscis, as it has the highest occluded detection ratio. If an object is occluded, it does not make sense to estimate its position. This indicates the advantage of the proposed approach in linking tracks under merged conditions, which produces the tracks comparable to manual "point and click" results.

To show the advantage of our method in fulfilling two sub tasks, we illustrate ten consecutive sample frames of the final tracking results in Fig. 12. This is an extreme case of merge condition. As the result of sub task 1 , the label of each BB is estimated. The correct labels are given in (e) with the help of user correction, even though they do not follow the ascending order we assumed. Given the reliably labeled BBs, the positions of proboscis tips in (a) (i) in merged BBs are estimated with an acceptable precision by our track linking approach. As the final outputs, three trajectories of tips are drawn on one of the video frames for visualization, as shown in Fig. 13.

Table 4

Comparison of our method with ground truth.

\begin{tabular}{llll}
\hline Object name & R-antenna & Proboscis & L-antenna \\
\hline Average position error (pixels) & $5.3 \pm 0.5$ & $8.3 \pm 3.2$ & $6.4 \pm 0.73$ \\
TCF & $0.93 \pm 0.03$ & $0.58 \pm 0.16$ & $0.95 \pm 0.03$ \\
Merged (\%) & $0.57 \pm 0.47$ & $13 \pm 5.1$ & $0.93 \pm 1.0$ \\
Occluded (\%) & $0.95 \pm 0.93$ & $14 \pm 2.4$ & $2.5 \pm 2.2$ \\
FN (\%) & $5.5 \pm 3.2$ & $2.0 \pm 2.2$ & $5.80 \pm 2.8$ \\
FP (\%) & $0.16 \pm 0.19$ & $0.00 \pm 0.00$ & $1.4 \pm 0.90$ \\
\hline
\end{tabular}

a

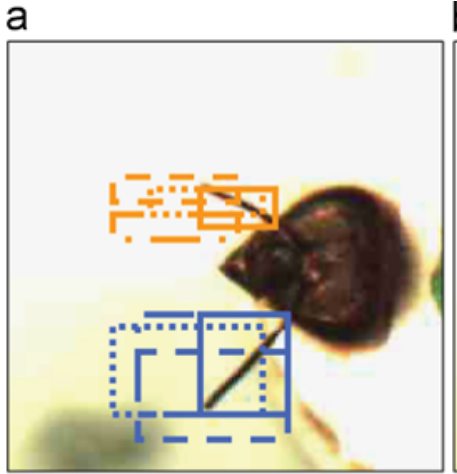

b

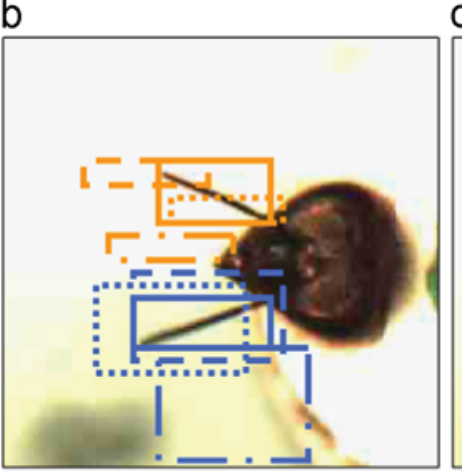

C

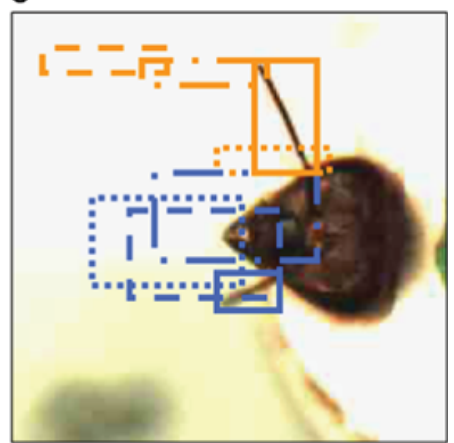

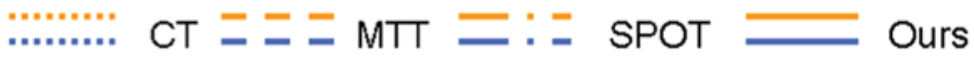

Fig. 11. Results of four tracking methods. (A colored version is available online.) 
a

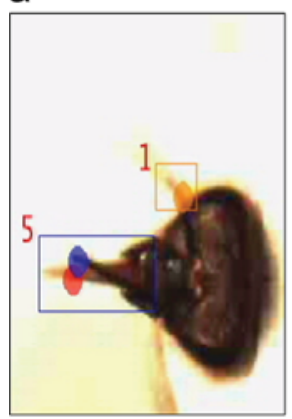

f

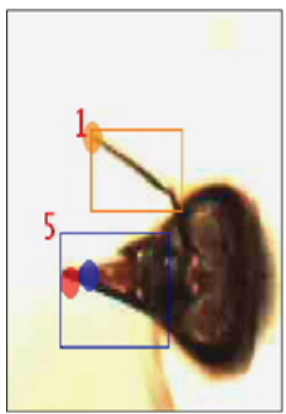

b

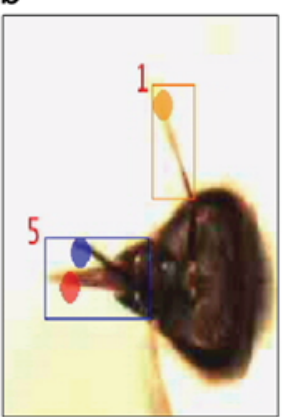

g

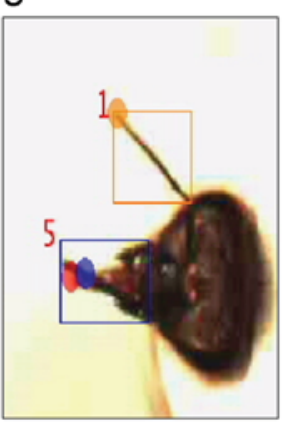

C

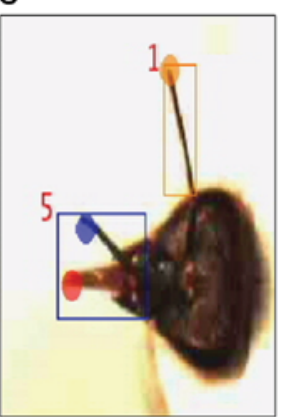

h

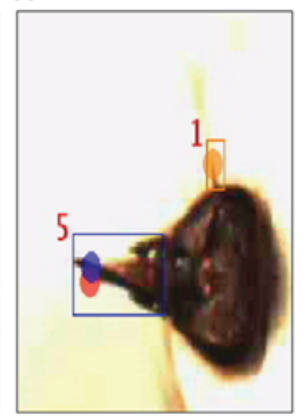

d

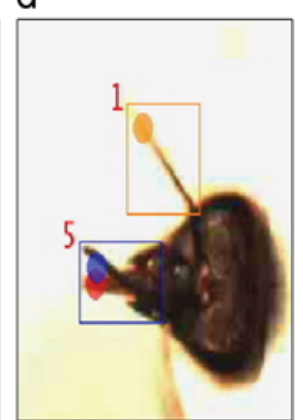

i

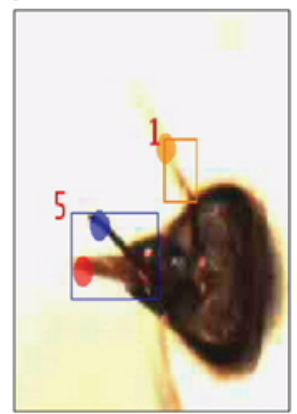

e

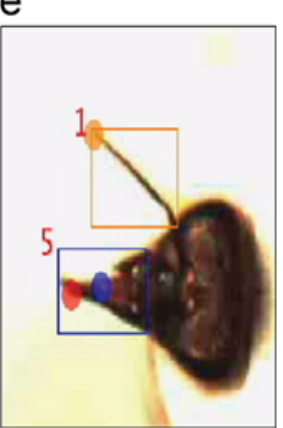

j

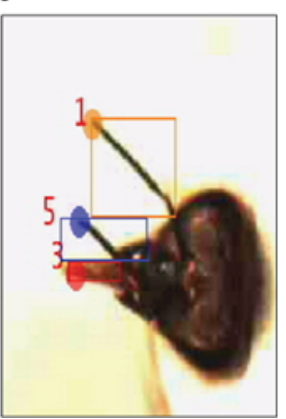

Fig. 12. Ten consecutive sample frames of the final tracking results under merge condition.

a

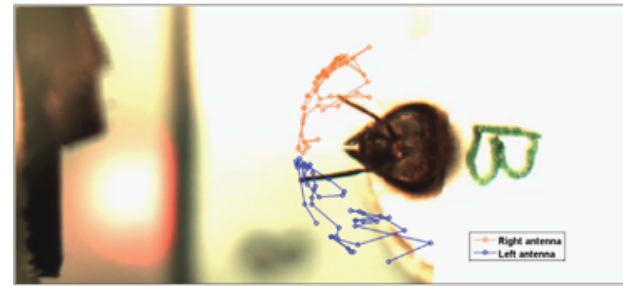

b

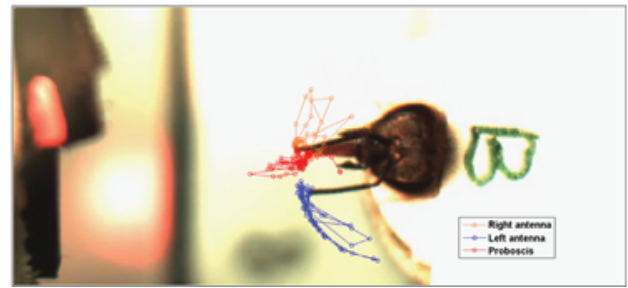

Fig. 13. Three trajectories of tips of 100 frame in the videos shown in Fig. 4 are drawn on one of the video frames: the orange dots denote the tips of right antenna, red for proboscis and blue for left antenna. (A colored version is available online.)

Table 5

Classification results in Section 4.1 for six shape descriptors.

\begin{tabular}{llllll}
\hline Shape descriptor & Antenna & Mandible & Proboscis & Mean & Variance \\
\hline EHD [43] & 0.96 & 0.66 & 0.55 & 0.72 & 0.05 \\
IEHD [44] & 0.16 & 0.43 & 0.95 & 0.51 & 0.16 \\
GF [45] & 0.99 & 0.34 & 0.67 & 0.67 & 0.11 \\
SSH [46] & 0.99 & 0.44 & 0.43 & 0.62 & 0.10 \\
FD [47] & 0.44 & 0.32 & 1.00 & 0.59 & 0.13 \\
ISH [44] & 0.99 & 0.33 & 0.47 & 0.60 & 0.12 \\
\hline
\end{tabular}

\subsubsection{Anatomical model}

To validate the analysis about the anatomical model of insect body parts in Section 3.2, we list the classification results of Section 4.1 for six shape descriptors in Table 5 tested on Bee videos. Similar to most biomedical data, the class distribution is skewed. For example, the number of mandibles is much smaller than antennae. The unbalanced data problem causes that the minority class is more likely to be misclassified than the majority class. Taking the unbalanced classes into account, the mean and variance are calculated by treating each class with equal weight. As shown in Table 5, EHD produces the highest mean value of classification results and the lowest variance, thus is selected for our work.

\section{Conclusion}

In this paper, we proposed a method aiming at achieving high precision of tracking multiple targets by minimizing additional human effort for correction. Our method integrates a frame query approach, enabling users to correct the erroneous tracking hypoth eses and making full use of the user input to optimize the final results. This is a preferable approach to traditional track and then rectification scheme, as it does not require an additional round of manual evaluation and correction while guaranteeing a high precision of the tracking results. Particularly, an important aspect of this system is its ability to estimate the trajectories of insect body parts at pixel precision even under merge conditions. The practicability and tracking performance of this system is validated on challenging video datasets for insect behavioral experiments.

\section{Conflict of interest}

None declared. 


\section{Acknowledgements}

The authors would like to thank Ci Wang for the helpful discussion on the paper, Le Duan, Manuel Wildner and Deepika Banakar for help with software development, testing and evalua tion, Oliver Kühn and Christopher Dieter Reinkemeier for the video acquisition, Cathrin Warnke for her proof reading. This work was funded by Bundesministerium für Bildung und Forschung (01GQ0931 to PS and CGG), with partial support also from the National Natural Science Foundation of China under Grant nos. 61302121, 61403182, and 61363046 .

\section{References}

[1] S. Sauer, M. Kinkelin, E. Herrmann, W. Kaiser, The dynamics of sleep-like behaviour in honey bees, J. Comp. Physiol. A 189 (8) (2003) 599-607.

[2] J. Erber, B. Pribbenow, A. Bauer, P. Kloppenburg, Antennal reflexes in the honeybee: tools for studying the nervous system, Apidologie 24 (1993) 238-296.

[3] V. Rehder, Quantification of the honeybee's proboscis reflex by electromyographic recordings, J. Insect Physiol. 33 (7) (1987) 501-507.

[4] B.H. Smith, C.I. Abramson, T.R. Tobin, Conditional withholding of proboscis extension in honeybees (apis mellifera) during discriminative punishment, J. Comp. Psychol. 105 (4) (1991) 345-356.

[5] M.A. Chabaud, J.M. Devaud, M.H. Pham-Delègue, T. Preat, L. Kaiser, Olfactory conditioning of proboscis activity in drosophila melanogaster, J. Comp. Physiol. A 192 (12) (2006) 1335-1348.

[6] F.J. Guerrieri, P. d'Ettorre, Associative learning in ants: conditioning of the maxilla-labium extension response in camponotus aethiops, J. Insect Physiol. 56 (1) (2010) 88-92.

[7] A. Pérez-Escudero, J. Vicente-Page, R.C. Hinz, S. Arganda, G.G.D. Polavieja, Idtracker: tracking individuals in a group by automatic identification of unmarked animals, Nat. Methods 11 (7) (2014) 743-748.

[8] H. Pistori, V.V.V.A. Odakura, J.B.O. Monteiro, W.N. Gonçalves, A.R. Roel, J.D.A. Silva, B.B. Machado, Mice and larvae tracking using a particle filter with an auto-adjustable observation model, Pattern Recognit. Lett. 31 (4) (2010) 337-346.

[9] M. Shen, W. Huang, P. Szyszka, C.G. Galizia, D. Merhof, Interactive framework for insect tracking with active learning, in: International Conference on Pattern Recognition, IEEE, 2014, pp. 2733-2738.

[10] W. Luo, X. Zhao, T.K. Kim, Multiple object tracking: a review, arXiv:1409.7618.

11] Y. Wu, J. Lim, M.H. Yang, Online object tracking: a benchmark, in: Conference on Computer Vision and Pattern Recognition, IEEE, 2013, pp. 2411-2418.

[12] Z. Oin, C.R. Shelton, Improving multi-target tracking via social grouping, in: Conference on Computer Vision and Pattern Recognition, IEEE, 2012, pp. 1972-1978.

[13] C. Huang, B. Wu, R. Nevatia, Robust object tracking by hierarchical association of detection responses, in: European Conference on Computer Vision, Springer, Marseille, France, 2008, pp. 788-801.

[14] B. Bose, X. Wang, E. Grimson, Multi-class object tracking algorithm that handles fragmentation and grouping, in: Conference on Computer Vision and Pattern Recognition, IEEE, 2007, pp. 1-8.

[15] A.G.A. Perera, C. Srinivas, A. Hoogs, G. Brooksby, W. Hu, Multi-object tracking through simultaneous long occlusions and split-merge conditions, in: Conference on Computer Vision and Pattern Recognition, vol. 1, IEEE, 2006 pp. 666-673.

[16] A. Veeraraghavan, R. Chellappa, M. Srinivasan, Shape-and-behavior encoded tracking of bee dances, Pattern Anal. Mach. Intell. 30 (3) (2008) 463-476.

[17] T. Landgraf, R. Rojas, Tracking honey bee dances from sparse optical flow fields, FB Math. Inform. FU (2007) 1-37.

[18] T. Balch, Z. Khan, M. Veloso, Automatically tracking and analyzing the behavio of live insect colonies, in: International Conference on Autonomous Agents, ACM, 2001, pp. 521-528.

[19] F. Ying, Visual ants tracking (Ph.D. thesis), University of Bristol, 2004.

[20] S. Mujagić, S.M. Würth, S. Hellbach, V. Dürr, Tactile conditioning and movement analysis of antennal sampling strategies in honey bees (Apis mellifera L.), J. Vis. Exp. 70 (2011) e50179.

[21] J. Voigts, B. Sakmann, T. Celikel, Unsupervised whisker tracking in unrestrained behaving animals, J. Neurophysiol. 100 (1) (2008) 504-515.

[22] B. Risse, S. Thomas, N. Otto, T. Löpmeier, D. Valkov, X. Jiang, C. Klämbt, Fim, a novel ftir-based imaging method for high throughput locomotion analysis, PloS one 8 (1) (2013) e53963.

[23] K. Branson, A.A. Robie, J. Bender, P. Perona, M.H. Dickinson, High-throughput ethomics in large groups of drosophila, Nat. Methods 6 (6) (2009) 451-457.
[24] L. Fiaschi, F. Diego, K. Gregor, M. Schiegg, U. Koethe, M. Zlatic, F.A. Hamprecht, Tracking indistinguishable translucent objects over time using weakly supervised structured learning, in: Conference on Computer Vision and Pattern Recognition, IEEE, 2014, pp. 2736-2743.

[25] A. Yao, J. Gall, C. Leistner, L.V. Gool, Interactive object detection, in: Conference on Computer Vision and Pattern Recognition, IEEE, 2012, pp. 3242-3249.

[26] J. Yuen, B. Russell, C. Liu, A. Torralba, Labelme video: building a video database with human annotations, in: International Conference on Computer Vision, IEEE, 2009, pp. 1451-1458.

[27] A. Buchanan, A. Fitzgibbon, Interactive feature tracking using kd trees and dynamic programming, in: Conference on Computer Vision and Pattern Recognition, vol. 1, IEEE, 2006, pp. 626-633.

[28] C. Vondrick, D. Ramanan, Video annotation and tracking with active learning, Neural Inf. Process. Syst. (2011) 28-36.

[29] C. Vondrick, D. Ramanan, D. Patterson, Efficiently scaling up video annotation with crowdsourced marketplaces, in: European Conference on Computer Vision, Springer, Heraklion, Crete, Greece, 2010, pp. 610-623.

[30] P. Kaewtrakulpong, R. Bowden, An improved adaptive background mixture model for realtime tracking with shadow detection, in: 2nd European Workshop on Advanced Video-Based Surveillance Systems, Springer, London, UK, 2001, pp. 135-144.

[31] D.S. Pham, O. Arandjelović, V. Svetha, Detection of dynamic background due to swaying movements from motion features, Image Process. 24 (1) (2015) 332-344.

[32] M. Shen, P. Szyszka, C.G. Galizia, D. Merhof, Automatic framework for tracking honeybee's antennae and mouthparts from low frame rate video, in: International Conference on Image Processing, IEEE, 2013, pp. 4112-4116.

[33] M. Shen, P. Szyszka, O. Deussen, C.G. Galizia, D. Merhof, Automated tracking and analysis of behavior in restrained insects, J. Neurosci. Methods 239 (2015) 194-205.

[34] Y. Huang, I. Essa, Tracking multiple objects through occlusions, in: Conference on Computer Vision and Pattern Recognition, IEEE, 2005, pp. 1051-1058.

[35] K. Zhang, L. Zhang, M.H. Yang, Real-time compressive tracking, in: European Conference on Computer Vision, Springer, Florence, Italy, 2012, pp. 864-877.

[36] D.G. Lowe, Distinctive image features from scale-invariant keypoints, Int. J. Comput. Vis. 60 (2) (2004) 91-110.

[37] R. Martin, O. Arandjelović, Multiple-object tracking in cluttered and crowded public spaces, Adv. Vis. Comput., Springer, Las Vegas, NV, USA (2010) 89-98.

[38] O. Arandjelović, Contextually learnt detection of unusual motion-based behaviour in crowded public spaces, in: Computer and Information Sciences II, Springer, London, UK, 2012, pp. 403-410.

[39] B.D. Lucas, T. Kanade, An iterative image registration technique with an application to stereo vision, in: International Joint Conference on Artificial Intelligence, vol. 2, 1981, pp. 674-679.

[40] C. Harris, M. Stephens, A combined corner and edge detector, in: Alvey Vision Conference, Manchester, UK, 1988, pp. 147-151.

[41] R. Arandjelović, A. Zisserman, Smooth object retrieval using a bag of boundaries, in: International Conference on Computer Vision, IEEE, 2011, pp. 375-382.

[42] O. Arandjelović, Gradient edge map features for frontal face recognition under extreme illumination changes, in: British Machine Vision Association Conference, Surrey, BMVA Press, Surrey, England, 2012, pp. 1-11.

[43] H. Frigui, P. Gader, Detection and discrimination of land mines in groundpenetrating radar based on edge histogram descriptors and a possibilistic Knearest neighbor classifier, Fuzzy Syst. 17 (1) (2011) 185-199.

[44] C. Li, K. Shirahama, M. Grzegorzek, Application of content-based image analysis to environmental microorganism classification, Int. J. Biocybern. Biomed. Eng. 35 (1) (2015) 10-21.

[45] S.Z. Li, Shape matching based on invariants, in: Shape Analysis, Progress in Neural Networks, Ablex, Norwood, NJ, 1999, pp. 203-228.

[46] D. Zhang, G. Liu, Review of shape representation and description techniques, Pattern Recognit. 37 (1) (2004) 1-19.

[47] D. Zhang, G. Lu, A comparative study of curvature scale space and fourier descriptors for shape-based image retrieval, J. Vis. Commun. Image Represent. 14 (1) (2003) 39-57.

[48] L. Zhang, Y. Li, R. Nevatia, Global data association for multi-object tracking using network flows, in: Conference on Computer Vision and Pattern Recognition, IEEE, 2008, pp. 1-8.

[49] G. Guo, C.R. Dyer, Learning from examples in the small sample case: face expression recognition, Syst. Man Cybern. Part B: Cybern. 35 (3) (2005) $477-488$.

[50] J. Munkres, Algorithms for assignment and transportation problems, J. Soc. Ind. Appl. Math. 5 (1) (1957) 32-38.

[51] A. Buchanan, A. Fitzgibbon, Zoo tracer, 〈http://research.microsoft.com/zootra cer $\rangle, 2014$.

[52] C.R. del Bianco, F. Jaureguizar, N. Garcia, Bayesian visual surveillance: a model for detecting and tracking a variable number of moving objects, in: International Conference on Image Processing, IEEE, 2011, pp. 1437-1440.

[53] A.I. Dell, J.A. Bender, K. Branson, I.D. Couzin, G.G.D. Polavieja, L.P.J.J. Noldus, A. Pérez-Escudero, P. Perona, A.D. Straw, M. Wikelski, Automated image-based tracking and its application in ecology, Trends Ecol. Evol. 29 (7) (2014) 417-428.

[54] T. Zhang, B. Ghanem, S. Liu, N. Ahuja, Robust visual tracking via multi-task sparse learning, in: Conference on Computer Vision and Pattern Recognition, IEEE, 2012, pp. 2042-2049. 
[55] L. Zhang, L.V.D. Maaten, Structure preserving object tracking, in: Conference on Computer Vision and Pattern Recognition, IEEE, 2013, pp. 1838-1845.
[56] Z. Kalal, J. Matas, K. Mikolajczyk, Pn learning: bootstrapping binary classifiers by structural constraints, in: Conference on Computer Vision and Pattern Recognition, IEEE, 2010, pp. 49-56.

Minmin Shen is currently a Postdoctoral Fellow at the Department of Computer and Information Science, University of Konstanz, Germany. She is also with School of Software Engineering, South China University of Technology. Her current research interests include biological image/video processing, computer vision, super-resolution, video coding, and video/image enhancement.

Chen Li got his Bachelor and Master degrees in University of Science and Technology Beijing, China and Northeast Normal University, China in 2008 and 2011, respectively. Currently, he is a PhD student in University of Siegen, Germany. His researching points are pattern recognition and microscopic image processing.

Wei Huang is an Associate Professor in the Nanchang University. He worked in the University of California San Diego, USA, and the Agency for Science Technology and Research, Singapore as a Research Staff. Dr. Huang recent research interests mainly include but not limited to medical image processing, pattern recognition, and computer vision.

Paul Szyszka is a researcher at the University of Konstanz. He uses behavioral assays and electro- and optophysiological recordings to study how insects perceive and learn odors in a turbulent and ever-changing olfactory environment.

Marcin Grzegorzek is an Assistant Professor at the University of Siegen heading the Pattern Recognition Group. His research interests include multimodal object recognition and scene analysis, semantic multimedia analysis and retrieval, as well as behavioral biometry and medical image processing. He is author and co-author of more than 70 papers.

Kimiaki Shirahama received his B.E., M.E. and D.E. degrees in Engineering from Kobe University, Japan in 2003, 2005 and 2011, respectively. Currently, he is a postdoctral researcher at Pattern Recognition Group in University of Siegen, Germany. His research interests include multimedia data processing, machine learning, data mining and virtual reality.

Dorit Merhof is a professor at the Institute of Imaging \& Computer Vision at RWTH Aachen University, Germany. She is a speaker of the GI special interest group "Visual Computing in Biology and Medicine". Her research interests include acquisition, processing and visualization of image data originating from various biomedical and industrial applications.

Oliver Deussen is a professor for Computer Graphics and Media Informatics at University of Konstanz, Germany, Visiting Professor at the Chinese Academy of Science in Shenzhen and serves as a Co-Editor in Chief of Computer Graphics Forum. His areas of interest are modeling and rendering of complex objects, Non-photorealistic rendering and Information Visualization. 